\title{
The COVID-19 Pandemic and Elimination of Tuberculosis in China
}

\author{
Daniel P. Chin ${ }^{*}$
}

One of the best kept secrets in global public health is how China achieved the tuberculosis (TB) targets in the United Nation's Millennium Development Goals (MDG). The MDG's TB targets were to reduce the prevalence and mortality of TB by $50 \%$ between 1990 and 2015. By 2010, China had reduced its TB prevalence and mortality by $65 \%$ and $80 \%,(1-2)$ which meant China exceeded the MDG targets 5 years before the MDG deadline. This impressive achievement helped China to move from a high to a medium TB-incidence country. Today, China still has the world's third highest number of new TB cases each year. But when adjusted for population size, it has the lowest TB incidence per capita among the 30 high TBburden countries (3).

In 2015, the global community committed to the 2030 Sustainable Development Goals (SDG), which included two new TB targets — reducing TB incidence and deaths by $80 \%$ and $90 \%$, respectively, compared to their 2015 levels (3). World Health Organization (WHO) went further by setting the 2035 END TB targets of reducing TB incidence and deaths by $90 \%$ and $95 \%$, respectively (3). WHO estimates that China's TB incidence in 2015 was around 65 cases per 100,000 population (4). A $90 \%$ reduction would bring $\mathrm{TB}$ incidence to less than 7 cases per 100,000 population, a level seen in most high-income countries. Achieving this will mean that China has eliminated TB as a major public health problem.

Although the SDG and END TB targets seem difficult to achieve, we can learn from how China achieved the MDG TB targets, which was made possible by China's renewed commitment to control major infectious diseases following the 2003 SARS epidemic (5). Today, we are in similar situation. China is once again impacted by the spread of another coronavirus. The country has been strengthening its health system to be more responsive to both current and future pandemics. Perhaps TB can once again benefit from the renewed focus on controlling infectious diseases. This commentary will discuss how China can apply the lessons and approaches from its COVID-19 response to make progress toward eliminating TB as a major public health problem.

\section{LEARNING FROM SUCCESSES IN TB CONTROL FOLLOWING THE SARS EPIDEMIC}

To achieve the MDG targets, China had to achieve an earlier set of global TB control targets: WHO's 2005 targets of finding $70 \%$ estimated TB cases and successfully treating $85 \%$ of them (5). Between 2000 and 2005, China implemented WHO's DOTS strategy nationwide through its CDC system (Table 1). The proportion of $\mathrm{TB}$ patients treated in the $\mathrm{CDC}$ system increased four-fold, and a much greater proportion of TB patients in China completed their treatment (I). This achievement was extremely important because, without this, China would not have achieved the MDG TB targets. In retrospect, among the 22 high TB-burden countries in 2005, China was the only country to achieve these WHO targets.

Three main factors contributed to the successes of TB control following the SARS epidemic (5). First and foremost, there was strong government commitment to improve the control of infectious diseases and reach the 2005 WHO TB targets. Governments at all levels were held accountable for 3 key TB targets: DOTS coverage, case-detection, and treatment success. Second, the government improved the public health system, including the development of an internet-based reporting system for notifiable infectious diseases. This greatly facilitated the reporting and follow-up of TB patients in the hospital system. Third, increased domestic and international resources were combined into a single plan focused on achieving the government targets.

\section{NEED TO IMPLEMENT A NEW TB CONTROL MODEL}

Despite the progress in controlling TB during the first decade after 2000, important challenges to the control of TB emerged during the second decade. First, drug-resistance surveillance studies in China documented a serious epidemic of multidrug-resistant 
TABLE 1. Evolution of tuberculosis (TB) control models and approaches to eliminate TB following the COVID-19 pandemic. DOTS strategy as basis for old TB Transition to new national TB control Post-pandemic acceleration toward TB control model (2001-2010) model (2011-2020) elimination (2021-2035)

TB control County/district CDC and township/ Designated hospitals for TB, county/ network village clinics form TB control network: - CDC: Responsible for diagnosis and treatment, reporting, and monitoring of township and village doctors in carrying out their TB control functions; traced TB suspects who did not come for evaluation after being referred; responsible for maintaining program quality and achieving program targets.

- Township and village clinics: Doctors referred TB suspects to CDC for evaluation, traced those who did not reach $\mathrm{CDC}$, and monitored patient's treatment in community.

- Hospitals: Required to report and refer TB suspects to CDC.

Technical approaches
Implemented in CDC clinics as DOTS strategy:

- Diagnosis: sputum smear microscopy and chest x-ray

- Treatment: Standard short-course chemotherapy with first-line TB drugs

- Management of treatment: Primarily provided by family members; some directly observed therapy, especially during intensive phase of treatment.

- TB surveillance system: internetbased disease reporting system allowed real-time reporting of TB suspects, and case-based electronic registry of notified TB cases. district CDC, and township/village clinics form 3-in-1 TB control network:

- Hospitals: Designated county/district hospitals provide diagnosis, treatment and reporting of routine TB patients; city/prefectural hospitals responsible for MDR/XDR-TB diagnosis and treatment. Other hospitals required to report and refer TB suspects to designated hospitals

- CDC: Responsible for monitoring of township and village doctors in carrying out their TB control functions; traced TB suspects who did not come for evaluation after being referred; monitor reporting by hospitals.

- Township and village clinics: Doctors refer TB suspects to hospitals for evaluation, trace those who did not reach hospitals, and monitor patient's treatment in community.

Implemented in hospitals according to national TB diagnosis and treatment guidelines:

- Diagnosis: CT scan and chest x-ray; smear microscopy, culture, and rapid molecular tests to detect $M$. tuberculosis and drug resistance.

- Treatment: Standard short-course chemotherapy with first-line TB drugs for drug-sensitive TB; second-line TB drug regimen for rifampin-resistant/ MDR TB. Bedaquiline introduced as a new TB drugs.

- Management of treatment: Primarily self-administered or monitoring by family members; use of digital adherence technologies.

- TB surveillance system: internetbased disease reporting system allowed real-time reporting of TB suspects, and case-based electronic registry of notified TB cases. Capture TB data directly from hospital medical information system.

- Use of the digital medium: Provide online training for health care workers, track TB patients using the medication monitor.
Strengthening of health system to address COVID-19 pandemic can help TB:

- Hospitals: Designated hospitals providing COVID-19 diagnosis and treatment will have the capabilities to treat complicated respiratory illnesses with improved infection control system; staff are more knowledgeable about respiratory infection control. Such capacities are now more decentralized down to county level and can improve TB treatment

- CDC: Capabilities to identify, trace, screen, and quarantine contacts are widely available. These can be used for TB contract investigation.

- Township and village clinics: Doctors are much more aware of respiratory symptoms and diseases and are on the look out for patients with respiratory symptoms. They can help look for TB patients.

Technical and programmatic approaches used in COVID-19 pandemic can apply to TB:

- Diagnosis: Large-scale network of molecular testing down to county level; laboratory network of genomic sequencing available. This can be used for rapid molecular testing for TB on a large scale, including for drug resistance.

- Treatment: Specific COVID-19 treatment guidelines provided to hospitals and implemented rapidly. TB treatment, including for MDR/XDR-TB, can be implement the same way.

- Contact investigation: Health care workers are trained to elicit contact information and better understand the environments facilitating airborne transmission; patients are much more aware of who they have been in contact with. Use of electronic surveillance have improved contact identification. TB can use this for contact tracing, testing, and treatment for LTBI.

- Large-scale screening and testing of COVID-19 in communities: Health departments and health care workers have experience from community screening programs; this can be used to implement active case-finding for TB.

- Large-scale COVID-19 vaccination in communities: Health departments and health care workers gain experience from vaccination programs; this can be used to implement TB vaccination programs for adults.

- Information system: Data on COVID19 cases quickly shared in real-time from health facilities to government and used to monitor pandemic. TB data from hospitals and other sources can be made available in real-time for monitoring. 
TABLE 1. (Continued)

\begin{tabular}{|c|c|c|c|}
\hline & $\begin{array}{l}\text { DOTS strategy as basis for old TB } \\
\text { control model (2001-2010) }\end{array}$ & $\begin{array}{c}\text { Transition to new national TB control } \\
\text { model }(2011-2020)\end{array}$ & $\begin{array}{c}\text { Post-pandemic acceleration toward TB } \\
\text { elimination }(2021-2035)\end{array}$ \\
\hline Funding & $\begin{array}{l}\text { Predominantly domestic funding but } \\
\text { with significant international } \\
\text { contribution; funding, mostly provided } \\
\text { from national level: } \\
\text { - Increasing amount of dedicated TB } \\
\text { funding by central government; } \\
\text { provincial and prefectural/county TB } \\
\text { funding also increased. } \\
\text { - Simple diagnosis and first-line TB } \\
\text { drugs provided largely free of charge. } \\
\text { Limited funds for township/village } \\
\text { doctors to carry out TB services. } \\
\text { - Funding from international } \\
\text { organizations (World Bank, DFID, } \\
\text { GFATM, JICA, CIDA, WHO) supported } \\
\text { scale-up of DOTS strategy. }\end{array}$ & $\begin{array}{l}\text { Entirely domestic funding, mostly } \\
\text { provided by provincial and local } \\
\text { governments: } \\
\text { - Dedicated TB funding by central } \\
\text { government for first-line drugs and } \\
\text { basic TB diagnosis; variable level of } \\
\text { dedicated TB funding from provincial } \\
\text { and local governments. } \\
\text { - National health insurance important } \\
\text { in paying for TB services provided by } \\
\text { hospitals (including diagnosis, } \\
\text { treatment, and hospitalization), but } \\
\text { patient out-of-pocket (OOP) payment } \\
\text { still substantial, especially for MDR- } \\
\text { TB. } \\
\text { - Government funding for township/ } \\
\text { village clinics to carry out public health } \\
\text { functions, including TB services. } \\
\text { - Supplemental funding provided by } \\
\text { some government sources to reduce } \\
\text { patient OOP expenses for TB services. }\end{array}$ & $\begin{array}{l}\text { Government funding to strengthen } \\
\text { pandemic preparedness and } \\
\text { responsiveness can enhance support } \\
\text { for TB: } \\
\text { - Funding to hospitals, CDC's, } \\
\text { laboratory network, primary health } \\
\text { care clinics, and health promotion can } \\
\text { all potentially benefit TB diagnosis and } \\
\text { treatment. } \\
\text { - In addition to government insurance, } \\
\text { government provided subsidies to } \\
\text { reduce out-of-pocket payment for } \\
\text { COVID-19 treatment. COVID-19 } \\
\text { vaccinations free of charge. These can } \\
\text { apply to provide entirely free diagnosis } \\
\text { and treatment or substantially reduced } \\
\text { cost of care for TB patients. }\end{array}$ \\
\hline
\end{tabular}

TB (MDR-TB) that was not being addressed (G). The CDC's were unable to diagnose or treat MDR-TB, and hospital treatment of TB was actually a risk factor for acquiring MDR-TB (6). Second, to focus the CDC system on its public health functions, the government began shifting $\mathrm{TB}$ diagnosis and treatment from the CDC's to the hospitals. This large-scale change carried certain risks because hospitals were not set up to provide community support to patients after they returned home to continue treatment, which often needed several weeks to months. A modeling study showed that a reduction in treatment quality from this shift could worsen the TB epidemic in China ( 7 ).

To address these challenges, a new $\mathrm{TB}$ control model was piloted by the China CDC under the guidance of the National Health Commission in collaboration with the Bill and Melinda Gates Foundation and then successfully implemented in 3 provinces (Table 1). The program had 3 important components. First, it fully incorporated the hospital system into the TB control network by designating county/district hospitals to diagnose and treat drugsensitive $\mathrm{TB}$ and city-level specialized hospitals to diagnose and treat MDR-TB. Second, it incorporated innovative approaches to modernize TB diagnosis and treatment. Third, it increased the coverage of national health insurance for TB diagnosis and treatment, which reduced catastrophic health expenses for TB patients, especially those with MDR-TB.

These 3 components worked synergistically to improve diagnosis and treatment for $\mathrm{TB}$, especially for MDR-TB (8). Take as an example a patient diagnosed with TB in the designated county hospital. His sputum specimens were transported to the designated city hospital, where rapid molecular testing revealed that he had rifampin-resistant TB. The information was quickly sent to staffs at the county hospital, and they quickly tracked down the patient and referred him to the city hospital for treatment. After being hospitalized and started on appropriate second-line TB drugs, he was referred back to the county $\mathrm{CDC}$, which arranged for regular follow-up by the township clinic. Although he was very poor, the rural health insurance scheme paid for a high percentage of his treatment cost, and he received additional subsidies from the Ministry of Civil Affair's subsidies for very poor rural residents. As a result, he was able to complete his two-year treatment course. This example illustrates the importance of having all three components of the model functioning together.

\section{HOW COVID-19 PANDEMIC CAN HELP ELIMINATE TB AS A PUBLIC HEALTH PROBLEM}

China is still far from the SDG and END TB targets. Since 2015, the decline in TB incidence has been approximately $2 \%$ per year (4). Improved treatment of drug-sensitive TB over the past two decades have substantially reduced new TB infections. But approximately $20 \%$ of China's population, or nearly 300 millon people, is already infected with $M$. tuberculosis (9), and TB from reactivation of latent TB 
infection (LTBI) will continues to develop in huge numbers each year. In fact, a modeling study suggested that the vast majority of China's TB cases are now arising from LTBI (10). Without new interventions to address LTBI, it will take several decades for China to eliminate TB. At the same time, MDR-TB will remains a serious problem. The proportion of new $\mathrm{TB}$ cases with rifampin-resistance or MDR have not declined appreciably since the first National TB Prevalence Survey in 2007 (3).

The COVID-19 pandemic provides a new opportunity to take rapid steps toward the SDG and END targets and eliminate TB and MDR-TB as a public health problem. The government has strengthened the health system to respond to the pandemic. It has also implemented new technical and programmatic approaches to keep the pandemic under control. In addition, there is major government funding to support pandemic preparedness and responsiveness. All of these can be leveraged for TB (Table 1). Specifically, they can support three key programmatic interventions needed to eliminate TB as a public health problem.

The first intervention is to fully transition all provinces to the new TB control model described above (Table 1). For instance, many cities and counties still do not have the diagnostic network for rapid molecular testing of $M$. tuberculosis and drug resistance, the quality of MDR-TB treatment is not uniform, follow-up of these patients during outpatient treatment is suboptimal, and designated hospitals are not properly staffed for $\mathrm{TB}$ or have poor infection control standard. Many of the pandemic-related improvements can help China implement the new TB control model and build a robust system of $\mathrm{TB}$ diagnosis and treatment for years to come.

The second intervention is to scale-up use of TB preventive treatment (TPT). If China is to substantially accelerate the decline in its TB incidence, it must address the large reservoir of LTBI in its population. The most cost-effective approach is to target the population most at risk for progressing from LTBI to active TB - close contacts of active TB cases (11). Finding and evaluating close contacts frequently lead to additional TB cases. But the full benefit of contact investigation comes from providing TPT to the contacts with TB infection (12).

Up to now, TB contact investigation and provision of TPT to close contacts have not been effectively implemented in high TB burden countries. Part of this is due to the implementation challenges. Current losses along the cascade to screen and treat individuals for LTBI is huge (13), raising the question of whether the benefits are worth the cost. But China has gained considerable experience with tracing and testing of COVID-19 contacts. This could be applied to TB. Shorter drug regimens - down to one month or 12 weekly doses - will make TPT much more feasible to implement $(12,14)$.

The third intervention is mass administration of new $\mathrm{TB}$ vaccines when they become available. There are two promising vaccination approaches. First, a study showed that BCG revaccination of adolescents reduced the risk of $M$. tuberculosis infection by $45 \%$ (15). A larger clinical trial is now underway to confirm this observation in South Africa with results anticipated by 2024 or 2025 . Second, a study of GSK's M72 vaccine demonstrated a $50 \%$ protection against progression to pulmonary TB in adults with LTBI (16). Planning for a phase 3 efficacy study is underway and the trial will likely begin early 2023 with results anticipated around 2028.

If one or both vaccine trials show efficacy in protection against $\mathrm{TB}$ infection or disease, vaccines will become important tools in the fight against TB. For China, with its high burden of LTBI, the availability of a vaccine that can prevent disease among latently infected persons will be especially important. Experience with rolling out COVID-19 vaccines in all age groups will help prepare for the rollout of new $\mathrm{TB}$ vaccines.

\section{ELIMINATION OF TB AS A CRITICAL PART OF THE HEALTH SECURITY AGENDA}

With the COVID-19 pandemic, the control of infectious diseases is once again high on the health and development agenda of the Chinese government. The government clearly recognizes the need to build the system and capabilities to respond rapidly and effectively to new epidemics. But the track record of governments around the world to prepare for new pandemics is poor. Following the 2009 H1N1 pandemic, an independent assessment found that the world was ill-prepared to respond to a global public health emergency (17). An assessment done prior to the COVID-19 pandemic found that most countries did not have sufficient capacity to detect and respond to a major epidemic (18).

Because many years could lapse between major pandemics, it is difficult to build and maintain a 
responsive and prepared system to deal with a pandemic when it does occur. The main capabilities needed to be built and maintained are those needed to rapidly detect and treat an airborne pathogen and prevent its spread. These system and capabilities are precisely the ones needed to eliminate $\mathrm{TB}$. Therefore, it makes sense to advocate for TB to be high on the health security agenda. The interventions needed to eliminate TB could be presented as the best way to build and maintain the health system's preparedness and responsiveness to a new pandemic.

Health security for China can work in synergy with the elimination of $\mathrm{TB}$ in the country. By implementing the recommended $\mathrm{TB}$ elimination interventions in this commentary as part of the government's health security agenda, China will be much closer to the SDG TB targets by 2030, and this could set the stage for China to be the first high TB burden country to eliminate $\mathrm{TB}$ as a public health problem by 2035 or soon thereafter.

doi: $10.46234 / \mathrm{ccdcw} 2021.069$

* Corresponding author: Daniel P. Chin, Daniel.Chin@gates foundation.org.

Submitted: March 10, 2021; Accepted: March 12, 2021

\section{REFERENCES}

1. Wang LX, Zhang H, Ruan YZ, Chin DP, Xia YY, Cheng SM, et al. Tuberculosis prevalence in China, 1990-2010; a longitudinal analysis of national survey data. Lancet 2014;383(9934):2057 - 64. http://dx. doi.org/10.1016/S0140-6736(13)62639-2).

2. Zhang H, Huang F, Chen W, Du X, Zhou MG, Hu J, et al. Estimates of tuberculosis mortality rates in China using the disease surveillance point system, 2004-2010. Biomed Environ Sci 2012;25(4):483-8. http://dx.doi.org/10.3967/0895-3988.2012.04.015.

3. World Health Organization. Global tuberculosis report 2020. Geneva: World Health Organization; 2020. License: CC BY-NC-SA 3.0 IGO. https://apps.who.int/iris/handle/10665/336069?search-result=true\&query Global+tuberculosis+report+2020\&scope $=\&$ rpp $=10 \&$ sort_by=score\&o rder=desc. [2021-3-3].

4. WHO. WHO TB burden estimates. https://www.who.int/teams/globaltuberculosis-programme/data. [2021-3-3].

5. Wang LD, Liu JJ, Chin DP. Progress in tuberculosis control and the evolving public-health system in China. Lancet 2007;369(9562):691 6. http://dx.doi.org/10.1016/S0140-6736(07)60316-X.
6. Zhao YL, Xu SF, Wang LX, Chin DP, Wang SF, Jiang GD, et al. National survey of drug-resistant tuberculosis in China. N Engl J Med 2012;366:2161 - 70. http://dx.doi.org/10.1056/NEJMoa1108789.

7. Lin $\mathrm{HH}$, Wang LX, Zhang $\mathrm{H}$, Ruan YZ, Chin DP, Dye C. Tuberculosis control in China: use of modelling to develop targets and policies. Bull World Health Organ 2015;93(11):790-8. http://dx.doi.org/10.2471/BLT.15.154492.

8. Li RZ, Ruan YZ, Sun Q, Wang XX, Chen MT, Zhang H, et al. Effect of a comprehensive programme to provide universal access to care for sputum-smear-positive multidrug-resistant tuberculosis in China: a before-and-after study. Lancet Glob Health 2015;3(4):e217- 28. http://dx.doi.org/10.1016/S2214-109X(15)70021-5.

9. Gao L, Lu W, Bai LQ, Wang XH, Xu JS, Catanzaro A, et al. Latent tuberculosis infection in rural China: baseline results of a populationbased, multicentre, prospective cohort study. Lancet Infect Dis 2015;15(3):310 - 9. http://dx.doi.org/10.1016/S1473-3099(14)710850.

10. Huynh GH, Klein DJ, Chin DP, Wagner BG, Eckhoff PA, Liu RZ, et al. Tuberculosis control strategies to reach the 2035 global targets in China: the role of changing demographics and reactivation disease. BMC Med 2015;13:88. http://dx.doi.org/10.1186/s12916-015-03414.

11. Lung T, Marks GB, Nhung NV, Anh NT, Le Phuong Hoa N, Anh LTN, et al. Household contact investigation for the detection of tuberculosis in Vietnam: economic evaluation of a cluster-randomised trial. Lancet Glob Health 2019;7(3):e376 - 84. http://dx.doi.org/10. 1016/S2214-109X(18)30520-5.

12. World Health Organization. Latent tuberculosis infection: updated and consolidated guidelines for programmatic management. Geneva: World Health Organization; 2018. License: CC BY-NC-SA 3.0 IGO. https://apps.who.int/iris/handle/10665/260233. [2021-3-3].

13. Alsdurf H, Hill PC, Matteelli A, Getahun H, Menzies D. The cascade of care in diagnosis and treatment of latent tuberculosis infection: a systematic review and meta-analysis. Lancet Infect Dis 2016;16 (11):1269 - 78. http://dx.doi.org/10.1016/S1473-3099(16)30216-X.

14. World Health Organization. Rapid Communication: on forthcoming changes to the programmatic management of tuberculosis preventive treatment. Geneva: World Health Organization; 2020. License: CC BY-NC-SA 3.0 IGO. https://www.who.int/tb/publications/2020/rapidcommunication-on-forthcoming-changes-to-tpt/en/. [2021-3-3].

15. Nemes E, Geldenhuys H, Rozot V, Rutkowski KT, Ratangee F, Bilek $\mathrm{N}$, et al. Prevention of $M$. tuberculosis infection with H4:IC31 Vaccine or BCG Revaccination. N Engl J Med 2018;379(2):138 - 49. http://dx.doi.org/10.1056/NEJMoa1714021.

16. Tait DR, Hatherill M, Van Der Meeren O, Ginsberg AM, Van Brakel E, Salaun B, et al. Final analysis of a trial of M72/AS01E vaccine to prevent tuberculosis. N Engl J Med 2019;381(25):2429 - 39. http://dx.doi.org/10.1056/NEJMoa1909953.

17. Fineberg HV. Pandemic preparedness and response-Lessons from the H1N1 Influenza of 2009. N Engl J Med 2014;370(14):1335 - 42. http://dx.doi.org/10.1056/NEJMra1208802.

18. Oppenheim B, Gallivan M, Madhav NK, Brown N, Serhiyenko V, Wolfe ND, et al. Assessing global preparedness for the next pandemic: development and application of an Epidemic Preparedness Index. BMJ Glob Health 2019;4(1):e001157. http://dx.doi.org/10.1136/bmjgh2018-001157.

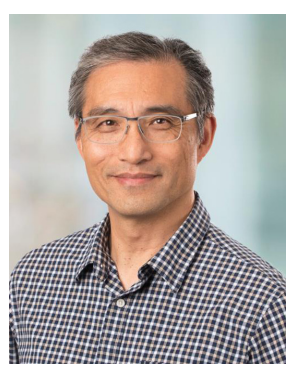

Daniel Chin, MD, MPH

Deputy Director, Global Health Program

Bill \& Melinda Gates Foundation 